\title{
Identification of key pathways and genes in endometrial cancer using bioinformatics analyses
}

\author{
YAN LIU, TENG HUA, SHUQI CHI and HONGBO WANG \\ Department of Obstetrics and Gynecology, Union Hospital, Tongji Medical College, \\ Huazhong University of Science and Technology, Wuhan, Hubei 430022, P.R. China
}

Received March 16, 2018; Accepted October 12, 2018

DOI: $10.3892 / \mathrm{ol} .2018 .9667$

\begin{abstract}
Endometrial cancer (EC) is one of the most common gynecological cancer types worldwide. However, to the best of our knowledge, its underlying mechanisms remain unknown. The current study downloaded three mRNA and microRNA (miRNA) datasets of EC and normal tissue samples, GSE17025, GSE63678 and GSE35794, from the Gene Expression Omnibus to identify differentially expressed genes (DEGs) and miRNAs (DEMs) in EC tumor tissues. The DEGs and DEMs were then validated using data from The Cancer Genome Atlas and subjected to gene ontology and Kyoto Encyclopedia of Genes and Genomes pathway analysis. STRING and Cytoscape were used to construct a protein-protein interaction network and the prognostic effects of the hub genes were analyzed. Finally, miRecords was used to predict DEM targets and an miRNA-gene network was constructed. A total of 160 DEGs were identified, of which 51 genes were highly expressed and 100 DEGs were discovered from the PPI network. Three overlapping genes between the DEGs and the DEM targets, BIRC5, CENPF and HJURP, were associated with significantly worse overall survival of patients with EC. A number of DEGs were enriched in cell cycle, human T-lymphotropic virus infection and cancer-associated pathways. A total of 20 DEMs and 29 miRNA gene pairs were identified. In conclusion, the identified DEGs, DEMs and pathways in EC may provide new insights into understanding the underlying molecular mechanisms that facilitate EC tumorigenesis and progression.
\end{abstract}

Correspondence to: Professor Hongbo Wang, Department of Obstetrics and Gynecology, Union Hospital, Tongji Medical College, Huazhong University of Science and Technology, 1277 Jiefang Avenue, Wuhan, Hubei 430022, P.R. China

E-mail: surgeonliuyan@163.com

Key words: endometrial cancer, bioinformatics analyses, differentially expressed genes, gene ontology, Kyoto Encyclopedia of Genes and Genomes enrichment analysis

\section{Introduction}

Endometrial carcinoma (EC) is one of the most common gynecological cancer types, with increasing global incidence in recent years (1). A total of 60,050 cases of EC and 10,470 EC-associated cases of mortality were reported in the USA in 2016 (1), which was markedly higher than the 2012 statistics of 47,130 cases and 8,010 mortalities (2). Although numerous studies have been conducted to investigate the mechanisms of endometrial tumorigenesis and development, to the best of our knowledge, the exact etiology remains unknown. Understanding the potential molecular mechanisms underlying EC initiation and progression is of great clinical significance. Previously, microarray technologies and bioinformatics have widely been used for the differential expression analysis of cancer and healthy cells to identify novel diagnostic and therapeutic biomarkers (3).

MicroRNAs (miRNAs) are small, noncoding RNAs that regulate the expression of critical genes involved in cancer progression and treatment (4). They bind to the 3'-untranslated region (3'-UTR) of target mRNAs (5), resulting in either degradation or inhibition of the expression and function of protein-coding mRNAs. miRNAs regulate several functions in cancer cells, including proliferation, apoptosis, metastasis, immune evasion and differentiation (6). In addition, several miRNAs serve critical roles in EC pathogenesis $(7,8)$ and are associated with clinicopathological features and survival (9). However, the specific mechanisms associated with miRNA-mediated regulation in EC require further investigation.

The current study evaluated the potential molecular mechanisms and biomarkers of EC using a bioinformatics approach. Microarray expression data were downloaded from the Gene Expression Omnibus (GEO) database and The Cancer Genome Atlas (TCGA). Differentially expressed genes (DEGs) and miRNAs (DEMs) in the EC samples compared with normal samples were identified using the GEO2R program and $R$ software. The DEGs were subjected to functional and pathway enrichment analysis, followed by protein-protein interaction (PPI) network and survival analysis. A putative miRNA-mRNA network relevant to EC pathogenesis was then constructed.

\section{Materials and methods}

Microarray expression data. The two gene expression datasets, GSE17025 (10) and GSE63678 (11), the miRNA 
expression dataset, GSE35794, and the DNA methylation profile, GSE40032, were downloaded from the GEO database (www.ncbi.nlm.nih.gov/geo). The GSE17025 dataset included data of 91 EC tissue samples, of which 79 were endometrioid and 12 were papillary serous, and 12 were atrophic endometrium samples from postmenopausal women. The tissue samples were analyzed on the GPL570 Platform Affymetrix Human Genome U133 Plus 2.0 (Affymetrix; Thermo Fisher Scientific, Inc., Waltham, MA, USA) (10). The GSE63678 dataset included data from seven EC tissues and five normal endometrium samples, and was analyzed on the GPL571 Platform Affymetrix Human Genome U133A 2.0 Array (Affymetrix; Thermo Fisher Scientific, Inc.) (11). The GSE35794 dataset included data from $18 \mathrm{EC}$ samples and four normal samples, and was analyzed on the GPL10850 Agilent-021827 Human miRNA Microarray V3 (Agilent Technologies, Palo Alto, CA, USA). The GSE40032 dataset included data of $64 \mathrm{EC}$ tissue samples and 23 normal endometrium samples, which was detected using the Illumina HumanMethylation27 BeadChip (HumanMethylation27_270596_v.1.2) on GPL8490 (Illumina, Inc., San Diego, CA, USA).

The RNA-seq ht seq-count data of mRNA, miRNA-seq and clinical data (project ID. TCGA-UCEC) of patients diagnosed with uterine corpus endometrial carcinoma were downloaded from TCGA (www.cancergenome.nih.gov) using the shengxin. ren download tool (http://www.shengxin.ren). Data of $552 \mathrm{EC}$ samples and 23 normal endometrium samples were included.

Identification of DEGs and DEMs. DEGs, DEMs and differentially methylated genes (DMGs) in the GSE17025, GSE35794 and GSE40032 datasets were identified using the GEO2R program of the GEO (www.ncbi.nlm.nih.gov/geo/geo2r/). The screening threshold of DEGs and DEMs was adjusted to $\mathrm{P}<0.05$ and $\log 2$ fold-change (FC) $\mid>1$. DMGs were identified with the thresholds of $\mathrm{P}<0.05$ and $|t|>2$, where $t$ is the ratio of the difference of the estimated value of a parameter from its hypothesized value to its standard error. For the dataset GSE63678, the original CEL files of the Affymetrix platform were background corrected, normalized and $\log 2$ transformed using the Robust Multi-array Average (RMA) (12) method and the affy package in R software (version 3.4.0; www.r-project. org). The Limma package (version 3.34.9) (13) was subsequently used for the calculation of aberrantly expressed mRNAs and the Benjamini-Hochberg (BH) method (14) was used to identify DEGs with the threshold criterion of $\mathrm{P}<0.05$ and absolute $\log 2 \mathrm{FC}>1$. The mRNA expression data of TCGA were calculated using Bioconductor package edgeR (version 3.20.9) (15) and were analyzed using the same strategy as used for the Affymetrix data analysis. The miRNA expression data of TCGA were analyzed using a Student's t-test in GraphPad Prism (version 6; GraphPad Software, Inc., La Jolla, CA, USA).

Gene ontology (GO) and Kyoto Encyclopedia of Genes and Genomes (KEGG) pathway enrichment analysis. GO and KEGG pathway enrichment analysis were performed to determine the biological significance of DEGs, using the Database for Annotation, Visualization and Integrated Discovery (DAVID; version 6.8; https://david.ncifcrf.gov/). The BH and Bonferroni methods were used for GO and pathway enrichment analysis.
PPI network and modular analysis. PPI networks are mathematical representations of physically interacting proteins (16). The STRING database (version 10.5; www.string-db.org) was used to establish the PPI network of DEGs and Cytoscape version 3.5 (17) was used to visualize the results. A confidence score $\geq 0.7$ was set as the cut-off criterion. Molecular Complex Detection (MCODE) was used to filter modules of the PPI network with a node score cut-off value of 0.2 , degree cut-off value of 2, $\mathrm{k}$-core of 2 and maximum depth of 100 (18).

Prediction of miRNA targets. The miRecords database (19) was used to predict the target genes of the DEMs. miRecords is a comprehensive database created using 11 established miRNA target prediction programs: MirTarget2, miTarget, MicroInspector, RNA22, PITA, miRanda, DIANA-microT, NBmiRTar, RNAhybrid, PicTar and TargetScan. Genes that were predicted by at least four programs were selected as the candidate targets of miRNAs.

Construction of the miRNA-target gene regulatory network. Overlaps between DEGs and DEM targets were selected and the association between overlapping genes and DEMs was validated using Pearson's correlation analysis in starBase (version 2.0; http://starbase.sysu.edu.cn/). The miRNA-gene regulatory network was constructed based on the overlapping genes and their upstream miRNAs, which were then visualized by Cytoscape software.

DEG survival analysis. OncoLnc (www.oncolnc.org) is a tool used for studying survival correlations by comparing clinical data with expression profiles of mRNAs, miRNAs and long non-coding RNAs (lncRNAs) (20). The overall survival (OS) rate of patients with EC relative to different DEGs was calculated using Kaplan-Meier analysis in OncoLnc. The associations between gene expression and clinical characteristics were analyzed using one-way ANOVA and a Bonferroni's multiple comparisons test in GraphPad Prism software.

\section{Results}

Identifying DEGs and DEMs. Gene expression profiles of EC and normal endometrium tissue datasets GSE17025 and GSE63678 were downloaded from GEO and normalized using the RMA method. The Limma package was used to analyze and compare the transcriptional data between EC samples and normal samples. Using $\mathrm{P}<0.05$ and absolute $\log 2 \mathrm{FC}>1$ as the cut-off criteria, 214 aberrantly expressed mRNAs were identified in EC (Fig. 1). A total of 205 identical DEGs were filtered from the two datasets, consisting of 131 upregulated and 74 downregulated genes that were similarly aberrantly expressed in the two datasets.

The TCGA RNA-seq data from 552 EC samples and 23 normal samples were normalized and corrected using the quantile normalization method and volcano plot analysis was performed using R software. A total of 7,562 aberrantly expressed mRNAs were obtained, of which 2,871 and 4,681 were upregulated and downregulated, respectively (Fig. 2). Finally, 160 aberrantly expressed genes, including 111 upregulated and 49 downregulated genes, were identified in EC samples from both the GEO and TCGA databases. The top 


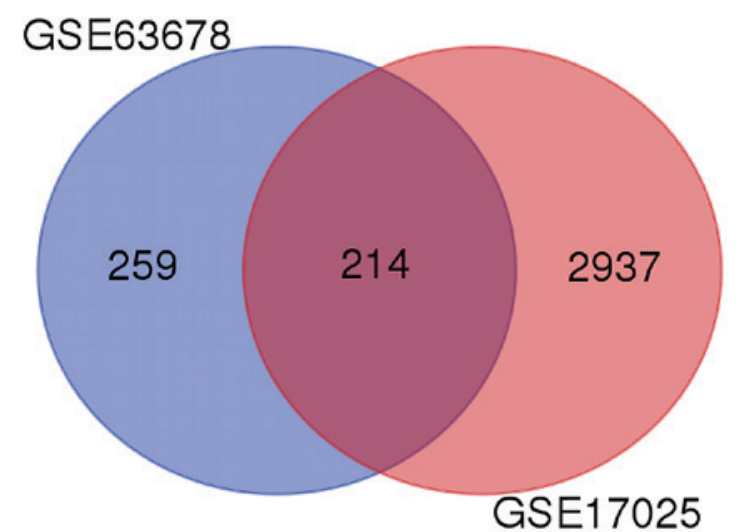

Figure 1. Identification of DEGs from the two datasets. The overlapping area corresponds to the commonly identified DEGs. $\mathrm{P}<0.05$ and $\mid \log$ (fold-change) $\mid>1$ indicated a statistically significant DEG. DEG, differentially expressed gene.

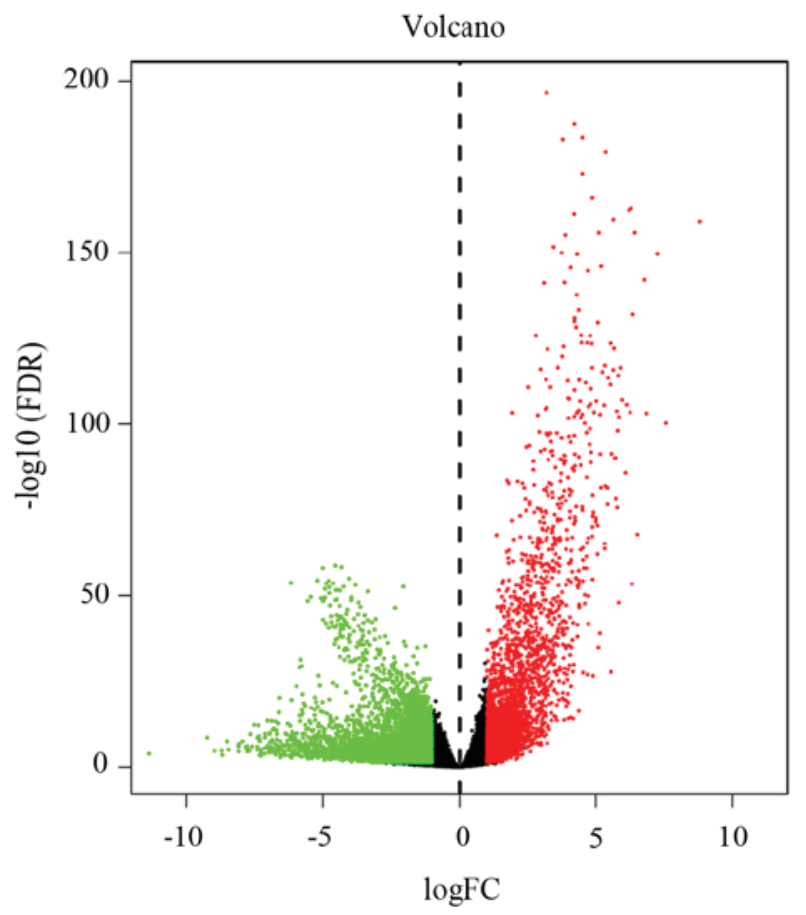

Figure 2. Volcano plot of detectable genome-wide mRNA profiles in 552 endometrial cancer tissue samples and 23 normal tissue samples. Red and green plots represent aberrantly expressed mRNAs with $\mathrm{P}<0.05$ and $|\log (\mathrm{FC})|>1$. Red plots indicate upregulated genes, green plots indicate downregulated genes and black plots indicate normally expressed mRNAs. The $\mathrm{x}$-axis is the fold-change value between the expression of circulating mRNAs in normal tissues and endometrial cancer tumors. The $y$-axis is the - $\log 10$ of the FDR value for each mRNA, representing the strength of the association. FDR, false discovery rate; FC, fold change.

ten DEGs identified between EC and normal tissue data from TCGA are presented in Table I.

For the dataset GSE40032, a total of 2,151 hypermethylated genes and 1,173 hypomethylated genes were identified using the cut-off criteria $\mathrm{P}<0.05$ and $|t|>2$. Subsequently, hypomethylation-high expression genes were obtained by overlapping hypomethylated and upregulated DEGs, and hypermethylation-low expression genes were obtained by overlapping hypermethylated and downregulated DEGs. A total
Table I. Top 10 differentially expressed genes in endometrial cancer compared with normal tissue according to data from The Cancer Genome Atlas.

\begin{tabular}{lcc} 
A, Upregulated genes & & \\
\hline DEG & $\log F \mathrm{C}$ & P-value \\
\hline SFN & 5.967256 & $1.35 \times 10^{-25}$ \\
UBE2C & 5.549047 & $7.32 \times 10^{-51}$ \\
CDC20 & 5.063798 & $1.02 \times 10^{-51}$ \\
HJURP & 5.004414 & $1.18 \times 10^{-60}$ \\
CENPA & 4.997543 & $7.26 \times 10^{-51}$ \\
BIRC5 & 4.996426 & $2.41 \times 10^{-45}$ \\
MELK & 4.883253 & $6.08 \times 10^{-56}$ \\
RRM2 & 4.797486 & $6.62 \times 10^{-43}$ \\
CDC45 & 4.797066 & $7.65 \times 10^{-57}$ \\
TPX2 & 4.748579 & $1.44 \times 10^{-49}$
\end{tabular}

B, Downregulated genes

\begin{tabular}{lcc}
\hline DEG & $\log$ FC & P-value \\
\hline BCHE & -5.68360 & $4.65 \times 10^{-93}$ \\
PEG3 & -5.53075 & $7.97 \times 10^{-115}$ \\
BNC2 & -4.43921 & $4.84 \times 10^{-105}$ \\
KIAA1644 & -4.23679 & $1.44 \times 10^{-74}$ \\
HAND2-AS1 & -3.59737 & $1.32 \times 10^{-41}$ \\
FGF2 & -3.52440 & $1.21 \times 10^{-67}$ \\
TRPC4 & -3.47055 & $1.19 \times 10^{-60}$ \\
TGFBR3 & -3.40246 & $4.98 \times 10^{-72}$ \\
AKT3 & -3.35874 & $4.21 \times 10^{-95}$ \\
SNCA & -3.25712 & $1.35 \times 10^{-56}$
\end{tabular}

DEG, differentially expressed gene; FC, fold-change.

of 12 hypomethylation-high expression genes (ESPL1, KIF14, KRT8, TYMS, SFN, TRIP13, S100A11, TK1, ASPM, CDCA3, CDCP1 and FUT2) and 15 hypermethylation-low expression genes (NAALAD2, RUNX1T1, TRPC4, TSPYL5, GPM6A, TCEAL2, ENPEP, ZFP2, PEG3, EFS, ST8SIA1, MAGEH1, CDO1, GSPT2 and FGF2) were obtained.

GO and KEGG pathway enrichment analysis of DEGs in $E C$. GO and KEGG enrichment analysis of the DEGs were conducted using DAVID. The DEGs were most highly enriched in biological processes associated with cell division, mitotic nuclear division and cell proliferation (Table II). According to KEGG pathway enrichment analysis, the DEGs were predominantly associated with cell cycle, human T-lymphotropic virus (HTLV-I) infection and pathways in cancer (Table III).

PPI network construction and modular analysis reveal critical candidate genes and pathways. STRING and Cytoscape software were used to screen 100 of the 160 DEGs into a PPI network complex, which contained 3,140 edges and 100 nodes (Fig. 3A). The remaining 60 DEGs did not fit into the PPI network. Of the 
Table II. GO enrichment analysis of differentially expressed genes in endometrial cancer.

\begin{tabular}{|c|c|c|c|c|}
\hline Term & Description & Count & P-value & FDR \\
\hline GO:0051301 & Cell division & 33 & $9.46131 \times 10^{-24}$ & $1.53 \times 10^{-20}$ \\
\hline GO:0007067 & Mitotic nuclear division & 23 & $3.07185 \times 10^{-16}$ & $5.32907 \times 10^{-13}$ \\
\hline GO:0005829 & Cytosol & 69 & $1.14723 \times 10^{-13}$ & $1.46927 \times 10^{-16}$ \\
\hline GO:0030496 & Midbody & 16 & $2.8856 \times 10^{-13}$ & $3.69671 \times 10^{-16}$ \\
\hline GO:0007062 & Sister chromatid cohesion & 15 & $2.92563 \times 10^{-13}$ & $4.71811 \times 10^{-16}$ \\
\hline GO:0005634 & Nucleus & 87 & $1.79532 \times 10^{-11}$ & $2.30007 \times 10^{-8}$ \\
\hline GO:0000070 & $\begin{array}{l}\text { Mitotic sister } \\
\text { chromatid segregation }\end{array}$ & 9 & $2.71519 \times 10^{-11}$ & $4.379 \times 10^{-8}$ \\
\hline GO:0000775 & $\begin{array}{l}\text { Chromosome, centromeric } \\
\text { region }\end{array}$ & 11 & $4.53211 \times 10^{-11}$ & $5.80631 \times 10^{-8}$ \\
\hline GO:0000777 & $\begin{array}{l}\text { Condensed chromosome } \\
\text { kinetochore }\end{array}$ & 12 & $1.89383 \times 10^{-10}$ & $2.42628 \times 10^{-7}$ \\
\hline GO:0000776 & Kinetochore & 11 & $1.65624 \times 10^{-9}$ & $2.12189 \times 10^{-6}$ \\
\hline GO:0000086 & $\begin{array}{l}\mathrm{G} 2 / \mathrm{M} \text { transition of } \\
\text { mitotic cell cycle }\end{array}$ & 13 & $2.80607 \times 10^{-9}$ & $4.52556 \times 10^{-6}$ \\
\hline GO:0005654 & Nucleoplasm & 54 & $3.75741 \times 10^{-9}$ & $4.81381 \times 10^{-6}$ \\
\hline GO:0005515 & Protein binding & 113 & $9.52955 \times 10^{-9}$ & $1.2931 \times 10^{-5}$ \\
\hline GO:0008283 & Cell proliferation & 18 & $2.22925 \times 10^{-8}$ & $3.59528 \times 10^{-5}$ \\
\hline GO:0000922 & Spindle pole & 11 & $3.06811 \times 10^{-8}$ & $3.9307 \times 10^{-5}$ \\
\hline GO:0000083 & $\begin{array}{l}\text { Regulation of transcription } \\
\text { involved in G1/S transition } \\
\text { of mitotic cell cycle }\end{array}$ & 7 & $3.62732 \times 10^{-8}$ & $5.85005 \times 10^{-5}$ \\
\hline GO:0005737 & Cytoplasm & 77 & $6.06599 \times 10^{-8}$ & $7.77145 \times 10^{-5}$ \\
\hline GO:0005876 & Spindle microtubule & 8 & $8.27121 \times 10^{-8}$ & 0.000105967 \\
\hline GO:0005819 & Spindle & 11 & $8.34624 \times 10^{-8}$ & 0.000106928 \\
\hline GO:0007059 & Chromosome segregation & 9 & $1.35128 \times 10^{-7}$ & 0.00021793 \\
\hline GO:0015630 & Microtubule cytoskeleton & 11 & $2.69353 \times 10^{-7}$ & 0.000345081 \\
\hline GO:0000082 & $\begin{array}{l}\text { G1/S transition of } \\
\text { mitotic cell cycle }\end{array}$ & 10 & $2.70795 \times 10^{-7}$ & 0.00043673 \\
\hline GO:0008017 & Microtubule binding & 13 & $3.46922 \times 10^{-7}$ & 0.000470751 \\
\hline GO:0031145 & $\begin{array}{l}\text { Anaphase-promoting } \\
\text { complex-dependent } \\
\text { catabolic process }\end{array}$ & 9 & $4.39782 \times 10^{-7}$ & 0.000709267 \\
\hline GO:0007080 & $\begin{array}{l}\text { Mitotic metaphase } \\
\text { plate congression }\end{array}$ & 7 & $7.55172 \times 10^{-7}$ & 0.001217916 \\
\hline GO:0005524 & ATP binding & 34 & $7.5564 \times 10^{-7}$ & 0.001025353 \\
\hline GO:0005874 & Microtubule & 14 & $2.64534 \times 10^{-6}$ & 0.003389028 \\
\hline GO:0005813 & Centrosome & 16 & $3.66421 \times 10^{-6}$ & 0.004694296 \\
\hline GO:0000281 & Mitotic cytokinesis & 6 & $4.8143 \times 10^{-6}$ & 0.007764095 \\
\hline GO:0005871 & Kinesin complex & 7 & $5.67045 \times 10^{-6}$ & 0.007264447 \\
\hline
\end{tabular}

GO, gene ontology; FDR, false discovery rate; ATP, adenosine 5'-triphosphate.

100 nodes, 57 hub genes were identified with a cut-off degree value of $>30$ and the top 10 genes with the most significant nodes were CDK1, CCNB1, CCNB2, TOP2A, CCNA2, CDC20, MAD2L1, BUB1B, NCAPG and CDCA8. According to the degree of importance, a significant module was selected from the PPI network complex for further analysis using MCODE. A total of 51 DEGs, including 51 nodes and 2,392 edges, were then selected as hub genes from the module (Fig. 3B).
Integrated network analysis of miRNA-mRNA interaction. A total of 35 DEMs were filtered from the GSE35794 dataset, of which 20, consisting of 14 upregulated and 6 downregulated miRNAs, were validated in TCGA data. As presented in Table IV, the most significantly upregulated miRNA was hsa-miR-200b, while the most significantly downregulated miRNA was hsa-miR-503. Subsequently, the predicted targets of DEMs were obtained on the basis of the miRecords 
Table III. Signaling pathway enrichment analysis of differentially expressed genes in endometrial cancer.

\begin{tabular}{llccc}
\hline ID & \multicolumn{1}{c}{ Term } & Count & P-value & FDR \\
\hline hsa04110 & Cell cycle & 17 & $7.65415 \times 10^{-14}$ & $9.04832 \times 10^{-11}$ \\
hsa04115 & p53 signaling pathway & 7 & $6.26158 \times 10^{-5}$ & 0.074039093 \\
hsa04914 & Progesterone-mediated oocyte & 7 & 0.000268953 & 0.317664885 \\
& maturation & & & 5.635320021 \\
hsa05166 & HTLV-I infection & 9 & 0.004891749 & 6.192004384 \\
hsa04114 & Oocyte meiosis & 6 & 0.005389396 & 7.171038253 \\
hsa01200 & Carbon metabolism & 6 & 0.006271196 & 18.59650509 \\
hsa05161 & Hepatitis B & 6 & 0.017244392 & 18.86329792 \\
hsa03460 & Fanconi anemia pathway & 4 & 0.017517104 & 21.35875474 \\
hsa05200 & Pathways in cancer & 10 & 0.020108452 & 23.69795919 \\
hsa01130 & Biosynthesis of antibiotics & 7 & 0.022606828 & 32.20200981 \\
hsa00010 & Glycolysis/Gluconeogenesis & 4 & 0.032322528 & 39.47199877 \\
hsa01230 & Biosynthesis of amino acids & 4 & 0.041557594 & \\
\hline
\end{tabular}

HTLV-1, human T-lymphotropic virus; FDR, false discovery rate.
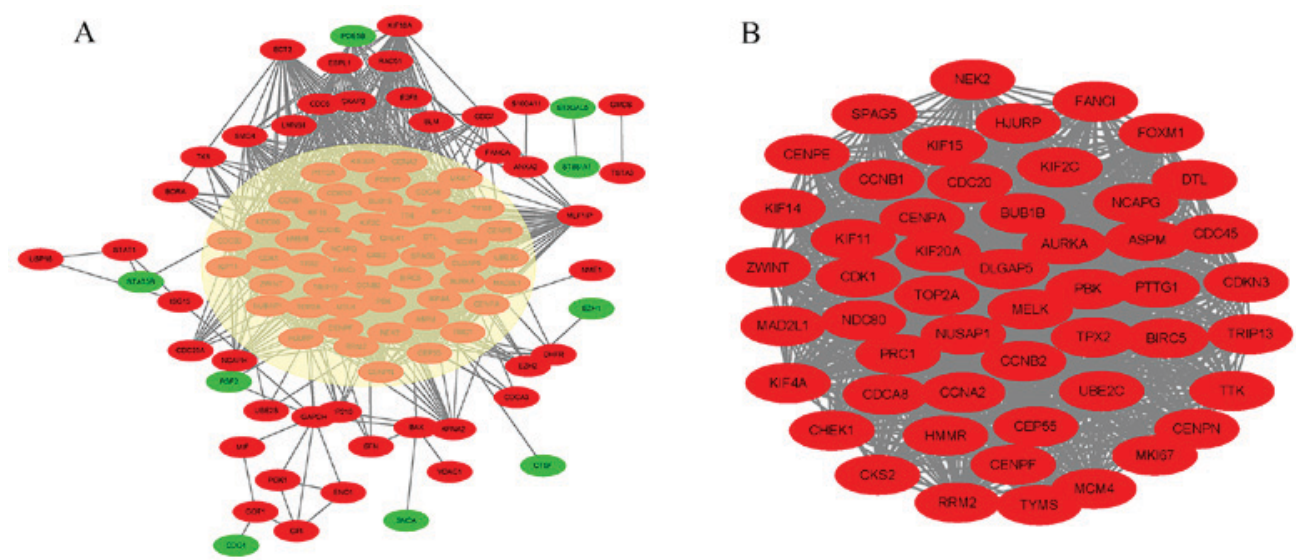

Figure 3. DEG PPI network and modular analysis. (A) Using the STRING online database, a total of 100 DEGs were filtered into the PPI network. The highlighted circle area is the most significant module. (B) The module consists of 51 nodes and 2,393 edges. DEG, differentially expressed gene; PPI, protein-protein interaction.

database. Since an inverse association was observed between miRNA expression and that of its target mRNA, DEMs with target genes identified as DEGs were selected for network analysis. A total of 29 pairs of DEMs and DEGs with an inverse association of expression met this criterion, including 14 DEMs and 14 overlapping genes (Fig. 4, Table V). Hsa-miR-203, hsa-miR-429, hsa-miR-200a, hsa-miR-200c and hsa-miR-141 exhibited the highest degrees (degree $\geq 3$ ) in the network (Table VI).

Survival analysis. The prognostic effects of the 51 hub genes in the PPI network were evaluated in OncoLnc. The OS of patients with EC was analyzed depending on low and high expression of each hub gene. TOP2A, CDCA8, AURKA, TTK, ASPM, CENPA, DLGAP5, RRM2, TPX2, KIF2C, UBE2C, CDC45, HMMR, FOXM1, KIF4A, TRIP13, SPAG5, MCM4, MKI67 and ESPL1 were significantly associated with worse OS (data not shown). The high mRNA expression levels of BIRC5, CENPF and HJURP were associated with worse OS of patients with EC (Fig. 5). In addition, BIRC5, CENPF and
HJURP were identified as target genes of the DEMs (Table V). Furthermore, the BIRC5 expression level was significantly associated with tumor grade $(\mathrm{P}<0.01)$, while CENPF and HJURP expression levels were significantly associated with high tumor grade and recurrence $(\mathrm{P}<0.05$; Fig. 6$)$.

\section{Discussion}

The incidence of EC and EC-associated mortality rate have been increasing in recent years despite improvements in surgical and chemo-therapies (1). Therefore, it is important to elucidate the potential mechanisms of EC tumorigenesis and development, and identify the key pathogenic factors to improve prognosis and clinical outcome.

The current study integrated two microarray expression profiles from GEO with TCGA data and identified 160 DEGs between the normal and tumor samples, including 111 upregulated and 49 downregulated genes. As per the GO and KEGG enrichment analysis, most of the DEGs were predicted to be associated with cell cycle, HTLV-I infection and pathways 
Table IV. Top five differentially expressed miRNAs in endometrial cancer compared with normal tissue.

A, Upregulated

\begin{tabular}{llc}
\hline miRNA & P-value & $\operatorname{logFC}$ \\
\hline hsa-miR-200b & 0.000101 & 7.633409 \\
hsa-miR-205 & 0.001261 & 7.413916 \\
hsa-miR-200a & 0.000101 & 7.382017 \\
hsa-miR-141 & 0.000143 & 7.254374 \\
hsa-miR-200c & 0.000143 & 7.108838 \\
\hline
\end{tabular}

B, Downregulated

\begin{tabular}{lcc}
\hline miRNA & P-value & $\operatorname{logFC}$ \\
\hline hsa-miR-503 & 0.027533 & -3.923641 \\
hsa-miR-876-3p & 0.047710 & -3.048536 \\
hsa-miR-144 & 0.043335 & -2.710278 \\
has-miR-133a & 0.000100 & -2.596223 \\
has-miR-154 & 0.000100 & -2.588022
\end{tabular}

miRNA or miR, microRNA; FC, fold-change.

in cancer. Following construction of the PPI network, 51 hub genes were identified. Similarly, 20 DEMs were identified from the GEO and TCGA databases. After integrating the target genes of these DEMs with the DEGs, 14 overlapping genes were identified, of which three hub genes (BIRC5, CENPF, HJURP) were associated with poor prognosis and aggressive grade of patients with EC.

The results of KEGG pathway analysis are noteworthy as several studies have previously demonstrated the involvement of the cell cycle in the development of EC $(21,22)$. HTLV-1 has been identified to cause specific $\mathrm{T}$ cell leukemias and lymphoma (23). HTLV-1 infection is also associated with other diseases, including neuroinflammatory disease (24), dermatitis (25) and uveitis (26). In some populations, the development of aggressive cervical carcinomas is associated with high HTLV-1 seroprevalence (27). In addition, certain cancer types have been associated with HTLV-1-hematologic malignancies (28), including adenocarcinoma of the thyroid or stomach and squamous cell carcinoma of the larynx, lip or lung. Notably, one previous study revealed the occurrence of endometrial adenocarcinoma in a rabbit inoculated with HTLV-1 (29). These findings are consistent with the current study, indicating an important role of the HTLV-1 infection pathway in EC.

miRNAs are a group of endogenous non-coding RNA molecules that can repress gene expression by targeting the 3'-UTR of mRNAs. Recent studies have reported that miRNA dysregulation may serve important roles in cancer development $(30,31)$. In the current study, 20 DEMs were identified in EC compared with normal tissues, including hsa-miR-203, hsa-miR-429, has-miR-200a, hsa-miR-200c and hsa-miR-141. Several studies have suggested that hsa-miR-203 not only

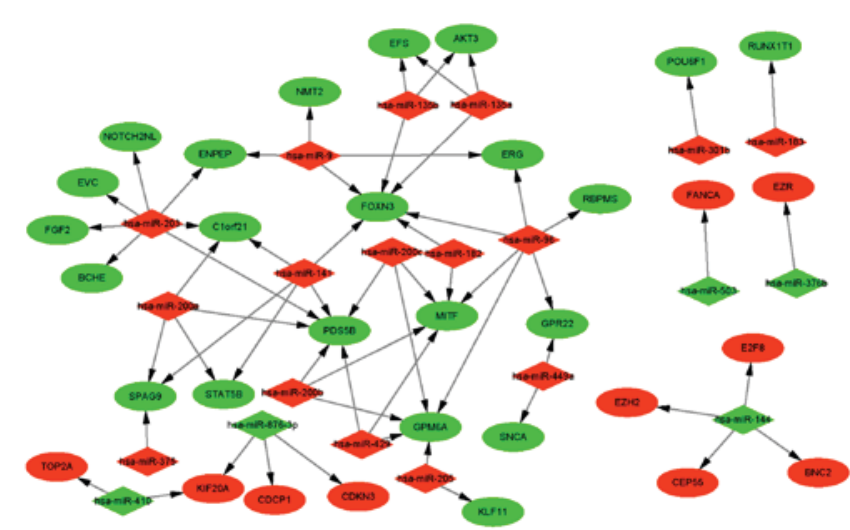

Figure 4. The miRNA-gene regulatory network in endometrial cancer. An ellipse represents a gene and a rhombus represents an miRNA. Red nodes represent upregulated genes and miRNAs, and green nodes represent downregulated genes and miRNAs in endometrial cancer. miRNA, micro-RNA.

functions as an oncogene, but also as a tumor suppressor. It is downregulated in several tumors, including non-small-cell lung cancer, gastric mucosa-associated lymphoid tissue lymphoma and myeloma, and can inhibit $\mathrm{G}$ protein signaling 17, as well as the oncogene, B-cell-specific Moloney murine leukemia virus insertion site-1 (32-34). As an oncogene, hsa-miR-203 is overexpressed in ovarian cancer tissues where it promotes glycolysis (35). One study has reported frequent hypermethylation of miR-203 in EC (36), however the expression of miR-203 was upregulated in the current study, consistent with the findings of Benati et al (37). miRNAs are regulated by multiple mechanisms including epigenetic, transcriptional, post-transcriptional and degradation regulation (38). Although it is reported that miR-203 hypermethylation is associated with EC, to the best of our knowledge, no studies have investigated the association between miR-203 hypermethylation and its expression level. The pathways of miR-203 upregulation in EC may be due to other mechanisms, which requires further investigation.

Hsa-miR-429 has been revealed to act as a tumor suppressor in renal cell carcinoma, gastric cancer and glioblastoma, by inhibiting cell proliferation, invasion and metastasis (39-41). However, hsa-miR-429 was upregulated in the current study, implying that it may function as an oncogene in EC. Hsa-miR-141 downregulates transmembrane-4-L-six-family-1 to inhibit pancreatic cancer cell invasion and migration and is widely considered as a potential candidate for the post-transcriptional regulation of phospholipase A2 receptor 1 expression in mammary cancer cells $(42,43)$. One study has demonstrated that hsa-miR-141 upregulation is important for EC growth (44). Based on the aforementioned findings, the current study hypothesizes that hsa-miR-203, hsa-miR-141 and hsa-miR-429 serve important roles in EC via different pathways.

Survival analysis of the overlapping DEGs and the target genes of the DEMs revealed that BIRC5, CENPF and HJURP were associated with poor prognosis of patients with EC. BIRC5 encodes survivin, which can regulate p21 expression in HeLa cells (45) and may be regulated by certain miRNAs $(45,46)$. Chuwa et al $(47)$ reported that a high 
Table V. Correlation between differentially expressed miRNAs and target genes.

\begin{tabular}{|c|c|c|c|c|c|}
\hline miRNA & Expression & Target gene & Expression & $\mathrm{r}$ & P-value \\
\hline hsa-miR-96 & Up & MITF & Down & -0.66790 & $3.77 \times 10^{-22}$ \\
\hline hsa-miR-449a & Up & SNCA & Down & -0.26599 & 0.00064878 \\
\hline hsa-miR-429 & Up & PDS5B & Down & -0.48758 & $5.39 \times 10^{-11}$ \\
\hline hsa-miR-429 & Up & MITF & Down & -0.55316 & $2.76 \times 10^{-14}$ \\
\hline hsa-miR-203 & Up & SPARC & Down & -0.39811 & $1.70 \times 10^{-7}$ \\
\hline hsa-miR-203 & Up & PDS5B & Down & -0.43891 & $5.75 \times 10^{-9}$ \\
\hline hsa-miR-203 & Up & FGF2 & Down & -0.42727 & $1.58 \times 10^{-8}$ \\
\hline hsa-miR-200c & Up & PDS5B & Down & -0.52345 & $1.05 \times 10^{-12}$ \\
\hline hsa-miR-200c & Up & MITF & Down & -0.61052 & $8.09 \times 10^{-18}$ \\
\hline hsa-miR-200c & Up & GPM6A & Down & -0.61152 & $6.92 \times 10^{-18}$ \\
\hline hsa-miR-200b & Up & PDS5B & Down & -0.46714 & $4.19 \times 10^{-10}$ \\
\hline hsa-miR-200b & $\mathrm{Up}$ & GPM6A & Down & -0.64805 & $1.51 \times 10^{-20}$ \\
\hline hsa-miR-200a & Up & STAT5B & Down & -0.61687 & $2.96 \times 10^{-18}$ \\
\hline hsa-miR-200a & Up & SPAG9 & Down & -0.45047 & $2.02 \times 10^{-9}$ \\
\hline hsa-miR-200a & Up & PDS5B & Down & -0.46840 & $3.71 \times 10^{-10}$ \\
\hline hsa-miR-200a & Up & C1orf21 & Down & -0.43953 & $5.44 \times 10^{-9}$ \\
\hline hsa-miR-182 & Up & MITF & Down & -0.69192 & $2.90 \times 10^{-24}$ \\
\hline hsa-miR-182 & Up & FOXN3 & Down & -0.60518 & $1.85 \times 10^{-17}$ \\
\hline hsa-miR-141 & $\mathrm{Up}$ & STAT5B & Down & -0.66087 & $1.44 \times 10^{-21}$ \\
\hline hsa-miR-141 & $\mathrm{Up}$ & SPAG9 & Down & -0.47761 & $1.49 \times 10^{-10}$ \\
\hline hsa-miR-141 & Up & PDS5B & Down & -0.51944 & $1.66 \times 10^{-12}$ \\
\hline hsa-miR-141 & Up & FOXN3 & Down & -0.58257 & $5.20 \times 10^{-16}$ \\
\hline hsa-miR-141 & Up & C1orf21 & Down & -0.42973 & $1.28 \times 10^{-8}$ \\
\hline hsa-miR-135b & Up & FOXN3 & Down & -0.64504 & $2.59 \times 10^{-20}$ \\
\hline hsa-miR-429 & Up & GPM6A & Down & -0.66256 & $1.05 \times 10^{-21}$ \\
\hline hsa-miR-136 & Down & BIRC5 & $\mathrm{Up}$ & -0.16483 & $3.67 \times 10^{-2}$ \\
\hline hsa-miR-133a & Down & CENPF & Up & -0.39548 & $2.08 \times 10^{-7}$ \\
\hline hsa-miR-144 & Down & BNC2 & Up & -0.19293 & 0.0142069 \\
\hline has-miR-154 & Down & HJURP & Up & -0.16526 & 0.0361743 \\
\hline
\end{tabular}

miRNA or miR, microRNA.

expression level of BIRC5 is associated with poor prognosis of EC, while Li et al (48) demonstrated that low expression levels of CENPF are associated with better overall survival of patients with bladder cancer. HJURP encodes holiday junction recognition protein, a centromeric histone chaperone involved in de novo histone $\mathrm{H} 3$ variant $\mathrm{CenH} 3$ recruitment and may regulate proliferation and apoptosis in bladder cancer cells by dysregulating the cell cycle and reactive oxygen species metabolism via the peroxisome proliferator-activated receptor $\gamma$-sirtuin 1 feedback loop (49). Hu et al (50) identified that the overexpression of HJURP predicts a poor prognosis of hepatocellular carcinoma.

In conclusion, the current study identified 160 DEGs and 20 DEMs in EC, and 14 DEGs were identified as target genes of the DEMs. Network analysis indicated a co-regulatory association between hsa-miR-203, hsa-miR-429 and hsa-miR-141, as well as the corresponding target mRNAs. These findings may improve understanding of the pathogenesis and the potential molecular mechanisms involved in EC, and assist with the identification of novel diagnostic and therapeutic biomarkers. However, the current study has
Table VI. Node-degree analysis of miRNA-mRNA interactions.

\begin{tabular}{lc} 
Node & Degre \\
\hline hsa-miR-141 & 5 \\
hsa-miR-200a & 4 \\
hsa-miR-200c & 3 \\
hsa-miR-203 & 3 \\
hsa-miR-429 & 3 \\
hsa-miR-200b & 2 \\
hsa-miR-182 & 2 \\
hsa-miR-96 & 1 \\
hsa-miR-449a & 1 \\
hsa-miR-144 & 1 \\
hsa-miR-135b & 1 \\
hsa-miR-136 & 1 \\
hsa-miR-133a & 1
\end{tabular}

miRNA or miR, microRNA. 
A

BIRC5

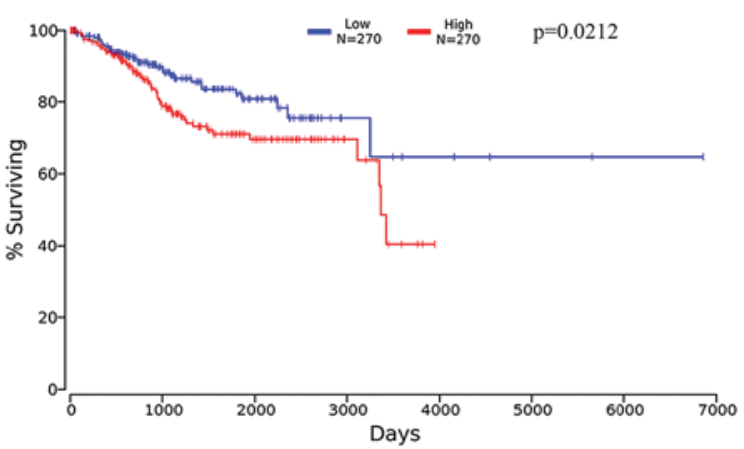

C

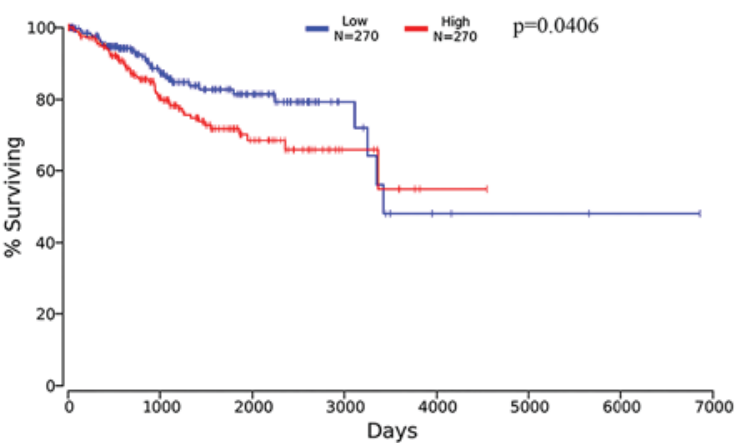

$\mathrm{B}$

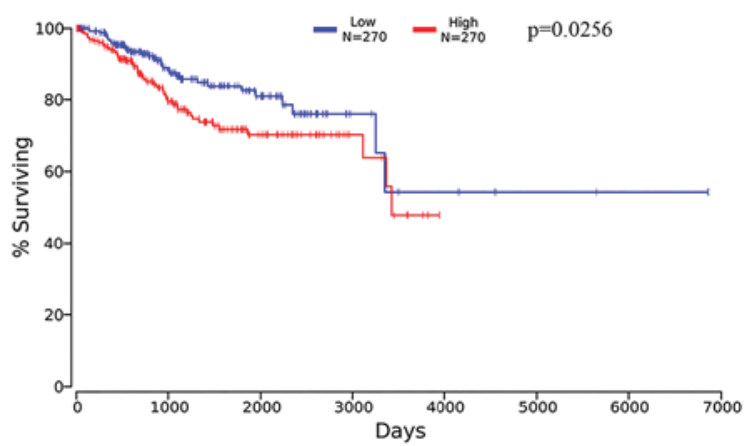

$\mathrm{D}$

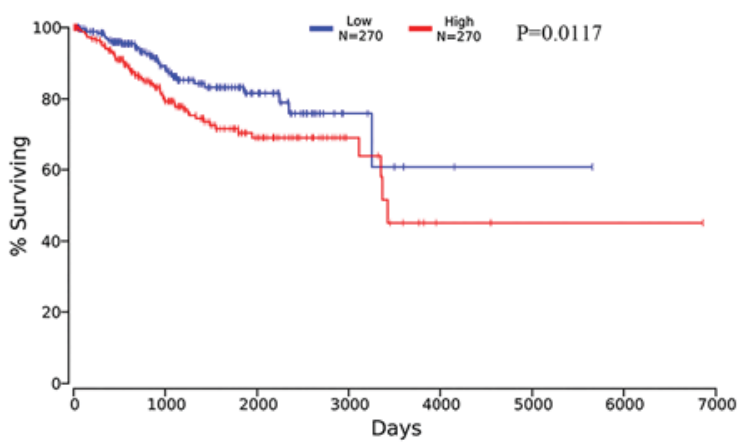

Figure 5. Kaplan-Meier curves for patients with endometrial cancer. The prognostic values of (A) BIRC5, (B) TOP2A, (C) CENPF and (D) HJURP were obtained by Kaplan-Meier analysis. These data were all from The Cancer Genome Atlas.
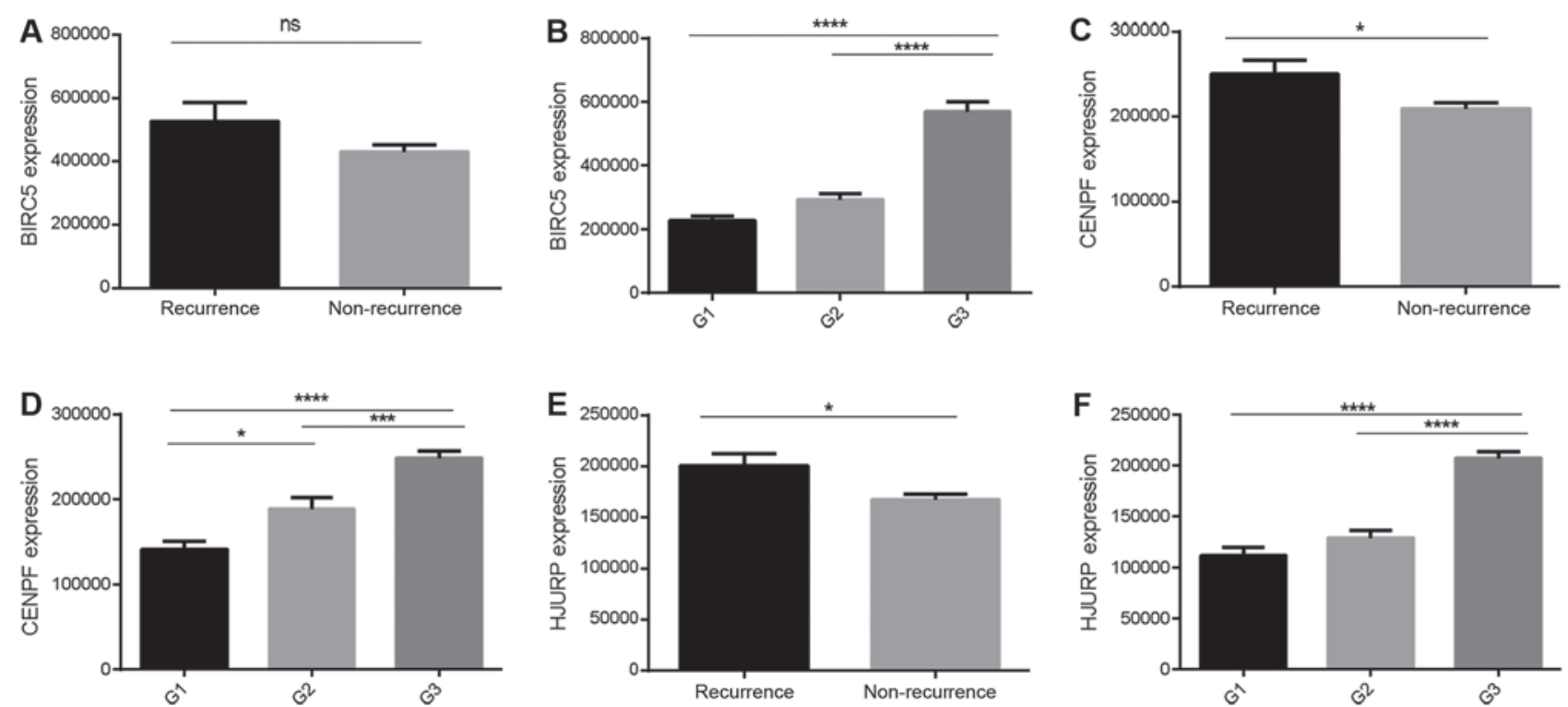

Figure 6. Associations between the expression levels of BIRC5, CENPF and HJURP, and clinical characteristics, including tumor grade and recurrence. (A and B) The association of BIRC5 expression level with tumor grade and recurrence. (C and D) The association of CENPF expression level with tumor grade and recurrence. (E and F) The association of HJURP expression level with tumor grade and recurrence. Data are presented as mean \pm the standard error of the mean. ${ }^{*} \mathrm{P}<0.05,{ }^{* *} \mathrm{P}<0.01,{ }^{* * *} \mathrm{P}<0.001,{ }^{* * * *} \mathrm{P}<0.0001$. ns, not significant.

limitations. The regulation of DEGs is complicated and the current study has only investigated the regulators of DEGs at the post-transcriptional level (miRNA) and the epigenetic level (DNA methylation). Additional studies should be performed to identify the putative regulators of DEGs. For example, future studies may construct a transcription factor-mRNA network to identify regulators at the transcriptional level. 


\section{Acknowledgements}

Not applicable.

\section{Funding}

Not applicable.

\section{Availability of data and materials}

The datasets used and analyzed during the present study are available from the corresponding author on reasonable request.

\section{Authors' contributions}

YL and HW conceived and designed the study; YL, TH, and SC performed data analysis; YL and $\mathrm{HW}$ wrote the manuscript.

\section{Ethics approval and consent to participate}

Not applicable.

\section{Patient consent for publication}

Not applicable.

\section{Competing interests}

The authors declare that they have no competing interests.

\section{References}

1. Siegel RL, Miller KD and Jemal A: Cancer statistics, 2016. CA Cancer J Clin 66: 7-30, 2016.

2. Siegel R, Naishadham D and Jemal A: Cancer statistics, 2012. CA Cancer J Clin 62: 10-29, 2012.

3. Vogelstein B, Papadopoulos N, Velculescu VE, Zhou S, Diaz LA $\mathrm{Jr}$ and Kinzler KW: Cancer genome landscapes. Science 339: $1546-1558,2013$

4. Mehrgou A and Akouchekian M: Therapeutic impacts of microRNAs in breast cancer by their roles in regulating processes involved in this disease. J Res Med Sci 22: 130, 2017.

5. Ambros V: The functions of animal microRNAs. Nature 431: 350-355, 2004

6. Croce CM and Calin GA: miRNAs, cancer, and stem cell division. Cell 122: 6-7, 2005

7. Dong P, Konno Y, Watari H, Hosaka M, Noguchi M and Sakuragi N: The impact of microRNA-mediated PI3K/AKT signaling on epithelial-mesenchymal transition and cancer stemness in endometrial cancer. J Transl Med 12: 231, 2014.

8. Ramón LA, Braza-Boïls A, Gilabert J, Chirivella M, España F, Estellés A and Gilabert-Estellés J: microRNAs related to angiogenesis are dysregulated in endometrioid endometrial cancer. Hum Reprod 27: 3036-3045, 2012.

9. Devor EJ, Miecznikowski J, Schickling BM, Gonzalez-Bosquet J, Lankes HA, Thaker P, Argenta PA, Pearl ML, Zweizig SL, Mannel RS, et al: Dysregulation of miR-181c expression influences recurrence of endometrial endometrioid adenocarcinoma by modulating NOTCH2 expression: An NRG oncology/gynecologic oncology group study. Gynecol Oncol 147: 648-653, 2017.

10. Day RS, McDade KK, Chandran UR, Lisovich A, Conrads TP, Hood BL, Kolli VS, Kirchner D, Litzi T and Maxwell GL: Identifier mapping performance for integrating transcriptomics and proteomics experimental results. BMC Bioinformatics 12: 213, 2011.

11. Pappa KI, Polyzos A, Jacob-Hirsch J, Amariglio N, Vlachos GD, Loutradis D and Anagnou NP: Profiling of discrete gynecological cancers reveals novel transcriptional modules and common features shared by other cancer types and embryonic stem cells PLoS One 10: e0142229, 2015.
12. Wilson CL and Miller CJ: Simpleaffy: A BioConductor package for affymetrix quality control and data analysis. Bioinformatics 21: 3683-3685, 2005.

13. Ritchie ME, Phipson B, Wu D, Hu Y, Law CW, Shi W and Smyth GK: limma powers differential expression analyses for RNA-sequencing and microarray studies. Nucleic Acids Res 43: e47, 2015

14. Hardcastle TJ: Generalized empirical Bayesian methods for discovery of differential data in high-throughput biology. Bioinformatics 32: 195-202, 2016.

15. Robinson MD, McCarthy DJ and Smyth GK: edgeR: A Bioconductor package for differential expression analysis of digital gene expression data. Bioinformatics 26: 139-140, 2010.

16. Szklarczyk D, Morris JH, Cook H, Kuhn M, Wyder S, Simonovic M, Santos A, Doncheva NT, Roth A, Bork P, et al: The STRING database in 2017: Quality-controlled protein-protein association networks, made broadly accessible. Nucleic Acids Res 45: D362-D368, 2017.

17. Shannon P, Markiel A, Ozier O, Baliga NS, Wang JT, Ramage D, Amin N, Schwikowski B and Ideker T: Cytoscape: A software environment for integrated models of biomolecular interaction networks. Genome Res 13: 2498-2504, 2003.

18. Bader GD and Hogue CW: An automated method for finding molecular complexes in large protein interaction networks. BMC Bioinformatics 4: 2, 2003.

19. Xiao F, Zuo Z, Cai G, Kang S, Gao X and Li T: miRecords: An integrated resource for microRNA-target interactions. Nucleic Acids Res 37: D105-D110, 2009.

20. Anaya J: OncoLnc: Linking TCGA survival data to mRNAs, miRNAs, and lncRNAs. PeerJ Computer Science 2: e67, 2016.

21. Suh DS, Park SE, Jin H, Lee K and Bae J: LRIG2 is a growth suppressor of Hec-1 A and Ishikawa endometrial adenocarcinoma cells by regulating PI3K/AKT- and EGFR-mediated apoptosis and cell-cycle. Oncogenesis 7: 3, 2018.

22. Shyam H, Singh N, Kaushik S, Sharma R and Balapure AK: Centchroman induces redox-dependent apoptosis and cell-cycle arrest in human endometrial cancer cells. Apoptosis 22: 570-584, 2017.

23. Nosaka K, Iwanaga M, Imaizumi Y, Ishitsuka K, Ishizawa K, Ishida Y, Amano M, Ishida T, Uike N, Utsunomiya A, et al: Epidemiological and clinical features of adult T-cell leukemia-lymphoma in Japan, 2010-2011: A nationwide survey. Cancer Sci 108: 2478-2486, 2017.

24. Matsuura E, Nozuma S, Tashiro Y, Kubota R, Izumo S and Takashima H: HTLV-1 associated myelopathy/tropical spastic paraparesis (HAM/TSP): A comparative study to identify factors that influence disease progression. J Neurol Sci 371: 112-116, 2016.

25. Lee R and Schwartz RA: Human T-lymphotrophic virus type 1-associated infective dermatitis: A comprehensive review. J Am Acad Dermatol 64: 152-160, 2011

26. Kamoi K and Mochizuki M: HTLV-1 uveitis. Front Microbiol 3 . 270, 2012

27. Strickler HD, Rattray C, Escoffery C, Manns A, Schiffman MH, Brown C, Cranston B, Hanchard B, Palefsky JM and Blattner WA: Human T-cell lymphotropic virus type I and severe neoplasia of the cervix in Jamaica. Int J Cancer 61: 23-26, 1995.

28. Imamura N, Inada $\mathrm{T}$, Tagaya $\mathrm{Y}$, Yodoi J and Kuramoto A: Association between ATL and non-hematopoietic neoplasms. Hematol Oncol 11: 127-137, 1993.

29. Zhao TM, Bryant MA, Kindt TJ and Simpson RM: Monoclonally integrated HTLV type 1 in epithelial cancers from rabbits infected with an HTLV type 1 molecular clone. AIDS Res Hum Retroviruses 18: 253-258, 2002.

30. Ma J, Li D, Kong FF, Yang D, Yang $\mathrm{H}$ and Ma XX: miR-302a-5p/367-3p-HMGA2 axis regulates malignant processes during endometrial cancer development. J Exp Clin Cancer Res 37: 19, 2018.

31. Srivastava SK, Ahmad A, Zubair H, Miree O, Singh S, Rocconi RP, Scalici J and Singh AP: MicroRNAs in gynecological cancers: Small molecules with big implications. Cancer Lett 407: 123-138, 2017.

32. Chi Y, Jin Q, Liu X, Xu L, He X, Shen Y, Zhou Q, Zhang J and Jin M: miR-203 inhibits cell proliferation, invasion, and migration of non-small-cell lung cancer by downregulating RGS17. Cancer Sci 108: 2366-2372, 2017.

33. Fernández C, Bellosillo B, Ferraro M, Seoane A, Sánchez-González B, Pairet S, Pons A, Barranco L, Vela MC, Gimeno E, et al: MicroRNAs 142-3p, miR-155 and miR-203 are deregulated in gastric MALT lymphomas compared to chronic gastritis. Cancer Genomics Proteomics 14: 75-82, 2017. 
34. Wu SQ, Niu WY,Li YP, Huang HB and Zhan R: miR-203 inhibits cell growth and regulates G1/S transition by targeting Bmi-1 in myeloma cells. Mol Med Rep 14: 4795-4801, 2016.

35. Xiaohong Z, Lichun F, Na X, Kejian Z, Xiaolan X and Shaosheng W: MiR-203 promotes the growth and migration of ovarian cancer cells by enhancing glycolytic pathway. Tumour Biol 37: 14989-14997, 2016.

36. Huang YW, Kuo CT, Chen JH, Goodfellow PJ, Huang TH, Rader JS and Uyar DS: Hypermethylation of miR-203 in endometrial carcinomas. Gynecol Oncol 133: 340-345, 2014

37. Benati M, Montagnana M, Danese E, Paviati E, Giudici S, Franchi M and Lippi G: Evaluation of mir-203 expression levels and DNA promoter methylation status in serum of patients with endometrial cancer. Clin Lab 63: 1675-1681, 2017.

38. Krol J, Loedige I and Filipowicz W: The widespread regulation of microRNA biogenesis, function and decay. Nat Rev Genet 11: 597-610, 2010.

39. Machackova T, Mlcochova H, Stanik M, Dolezel J, Fedorko M, Pacik D, Poprach A, Svoboda M and Slaby O: MiR-429 is linked to metastasis and poor prognosis in renal cell carcinoma by affecting epithelial-mesenchymal transition. Tumour Biol 37: 14653-14658, 2016.

40. Peng G, Liao Y and Shen C: miRNA-429 inhibits astrocytoma proliferation and invasion by targeting BMI1. Pathol Oncol Res 23: 369-376, 2017

41. Liu D, Xia P, Diao D, Cheng Y, Zhang H, Yuan D, Huang C and Dang C: MiRNA-429 suppresses the growth of gastric cancer cells in vitro. J Biomed Res 26: 389-393, 2012.

42. Xu L, Li Q, Xu D, Wang Q, An Y, Du Q, Zhang J, Zhu Y and Miao Y: Hsa-miR-141 downregulates TM4SF1 to inhibit pancreatic cancer cell invasion and migration. Int J Oncol 44: 459-466, 2014.

43. Menschikowski M, Hagelgans A, Nacke B, Jandeck C, Sukocheva O and Siegert G: Epigenetic control of phospholipase A2 receptor expression in mammary cancer cells. BMC Cancer 15: 971, 2015
44. Lee JW, Park YA, Choi JJ, Lee YY, Kim CJ, Choi C, Kim TJ, Lee NW, Kim BG and Bae DS: The expression of the miRNA-200 family in endometrial endometrioid carcinoma. Gynecol Oncol 120: 56-62, 2011

45. Xu Q, Liu M, Zhang J, Xue L, Zhang G, Hu C, Wang Z, He S, Chen L, Ma K, et al: Overexpression of KLF4 promotes cell senescence through microRNA-203-survivin-p21 pathway. Oncotarget 7: 60290-60302, 2016

46. Zhang D, Liu E, Kang J, Yang X and Liu H: MiR-3613-3p affects cell proliferation and cell cycle in hepatocellular carcinoma. Oncotarget 8: 93014-93028, 2017.

47. Chuwa AH, Sone K, Oda K, Ikeda Y, Fukuda T, Wada-Hiraike O, Inaba K, Makii C, Takeuchi M, Oki S, et al: Significance of survivin as a prognostic factor and a therapeutic target in endometrial cancer. Gynecol Oncol 141: 564-569, 2016.

48. Li S, Liu X, Liu T, Meng X, Yin X, Fang C, Huang D, Cao Y, Weng $H$, Zeng $X$ and Wang X: Identification of biomarkers correlated with the TNM staging and overall survival of patients with bladder cancer. Front Physiol 8: 947, 2017.

49. Cao R, Wang G, Qian K, Chen L, Qian G, Xie C, Dan HC, Jiang W, Wu M, Wu CL, et al: Silencing of HJURP induces dysregulation of cell cycle and ROS metabolism in bladder cancer cells via PPAR $\gamma$-SIRT1 feedback loop. J Cancer 8: 2282-2295, 2017.

50. Hu B, Wang Q, Wang Y, Chen J, Li P and Han M: Holliday junction-recognizing protein promotes cell proliferation and correlates with unfavorable clinical outcome of hepatocellular carcinoma. OncoTargets Ther 10: 2601-2607, 2017.

(i) $($ This work is licensed under a Creative Commons C. ${ }_{\text {EY NO NO }}$ Attribution-NonCommercial-NoDerivatives 4.0 International (CC BY-NC-ND 4.0) License. 